ARTICLE

https://doi.org/10.1038/s41467-019-12153-1

\title{
Surface association sensitizes Pseudomonas aeruginosa to quorum sensing
}

\author{
Sara K. Chuang1, Geoffrey D. Vrla ${ }^{2}$, Kathrin S. Fröhlich ${ }^{2,3}$ \& Zemer Gitai (iD ${ }^{2}$
}

In the pathogen Pseudomonas aeruginosa, LasR is a quorum sensing (QS) master regulator that senses the concentration of secreted autoinducers as a proxy for bacterial cell density. Counterintuitively, previous studies showed that saturating amounts of the LasR ligand, 30C12-HSL, fail to induce the full LasR regulon in low-density liquid cultures. Here we demonstrate that surface association, which is necessary for many of the same group behaviors as QS, promotes stronger QS responses. We show that lasR is upregulated upon surface association, and that surface-associated bacteria induce LasR targets more strongly in response to autoinducer than planktonic cultures. This increased sensitivity may be due to surface-dependent lasR induction initiating a positive feedback loop through the small RNA, Lrs1. The increased sensitivity of surface-associated cells to QS is affected by the type IV pilus (TFP) retraction motors and the minor pilins. The coupling of physical surface responses and chemical QS responses could enable these bacteria to trigger community behaviors more robustly when they are more beneficial.

\footnotetext{
${ }^{1}$ Department of Chemical and Biological Engineering, Princeton University, Princeton, NJ 08540, USA. ${ }^{2}$ Department of Molecular Biology, Princeton University, Princeton, NJ 08540, USA. ${ }^{3}$ Department of Biology I, Microbiology, Ludwig-Maximilians-University Munich, D-82152 Martinsried, Germany. Correspondence and requests for materials should be addressed to Z.G. (email: zgitai@princeton.edu)
} 
B acteria grow in a complex world and are constantly exposed to diverse stimuli. For many years the field has characterized the response of bacteria to the chemical stimuli of their environments, such as nutrients, compounds produced by other bacteria, or compounds produced by their hosts. For example, many bacteria use signal transduction mechanisms known as quorum sensing (QS) to monitor population density and regulate resource-intensive collective processes that would not benefit individual bacteria. These pathways typically involve the production of an autoinducer ligand, whose concentration is sensed by a transcriptional regulator ${ }^{1}$. More recently, increasing evidence suggests that bacteria also sense and respond to physical stimuli such as those associated with contacting a rigid surface. For example, in different species specific examples of biofilm formation $^{2}$, virulence induction ${ }^{3,4}$, swarming motility ${ }^{5}$, and twitching motility ${ }^{6}$ have each been shown to require bacteria to sense and respond to surface-association. QS and surface sensing have been implicated in regulating many of the same behaviors as described above, but to date have been considered largely independent processes $^{3,7,8}$.

In Pseudomonas aeruginosa, QS is mediated by a dense, interlinked network of to-date four known pathways: the IQS system, the quinolone system controlled by PqsR, and two LuxR/LuxI-type systems, LasR/LasI and RhlR/RhlI, respectively ${ }^{9}$. These systems are highly interconnected and LasR activation can induce a cascade that turns on the other systems as well ${ }^{9}$. When in complex with its autoinducer ligand, $\mathrm{N}$-(3oxododecanoyl)-homoserine lactone (3OC12-HSL), which is synthesized by $\operatorname{LasI}^{10}$, LasR functions as a transcriptional activator. One of the factors activated by 3OC12-HSL-bound LasR is the autoinducer synthase LasI, leading to the autoinduction canonical of QS circuits ${ }^{9,11-14}$. In addition, LasR is required for several surface-associated behaviors, including virulence induction, but was previously thought to function in parallel to surface sensing pathways ${ }^{3}$. Despite its importance at the top of the $P$. aeruginosa QS hierarchy, the transcriptional regulation of las $R$ itself is relatively poorly understood ${ }^{9,15}$. LasR induction has to date been primarily characterized in liquidgrown planktonic cultures. In these conditions, las $R$ transcript levels increase with cell density, but do so independently of the four characterized QS pathways $9,16,17$. Furthermore, addition of saturating amounts of exogenous 3OC12-HSL ligand is not sufficient to fully induce the LasR regulon, suggesting the presence of additional unknown LasR regulators ${ }^{14,18,19}$. Similarly, an analysis of multiple LasR-controlled genes indicated that most of these targets are not induced at low cell density even upon addition of $3 \mathrm{OC} 12-\mathrm{HSL}^{18}$. In many other systems, small RNAs (sRNAs) have emerged as QS regulators ${ }^{20}$, and LasR is known to regulate at least one sRNA, Lrs1 ${ }^{21}$. However, the regulation of LasR expression by sRNAs or surfaceassociation has not been examined.

Previous studies have implicated flagella, type IV pili (TFP), and PilY1 as candidate surface sensors. While flagella promote adhesion of $P$. aeruginosa in biofilm development in some contexts $^{22,23}$, virulence induction and biofilm formation share a common dependence on TFP. TFP form extracellular polymers that are actively extended and retracted by ATP-dependent motors and have been implicated as mechanosensors $4,24,25$ These polymers are generally thought to be composed of a major pilin subunit, PilA, extended by the motor PilB, and retracted by the motors PilT and PilU. With respect to virulence, TFP promote attachment to host cells ${ }^{26}$ and induce Vfr, a global regulator of virulence gene expression, in PAO1 strains ${ }^{4}$. In addition to PilA, $P$. aeruginosa also expresses the putative adhesin PilY1 and several minor pilin proteins with important structural and regulatory functions ${ }^{27}$. PilY1 is required for the ability of $P$. aeruginosa PA14 to kill amoebae host cells ${ }^{3}$, and has been suggested to function with TFP to promote biofilm formation 7,8 .

Here, we investigate the effect of surface-association on QS. We show that the QS master regulator LasR is upregulated upon surface-association, causing surface-associated cells to become more sensitive to the LasR ligand, 3OC12-HSL. Our data suggest a positive feedback system for increasing lasR expression that depends on the sRNA Lrs1, thereby allowing surface-associated cells to access QS-induced states that planktonic cells cannot. We also show that the increased QS sensitivity of surface-associated cells involves the TFP retraction motors and the minor pilins PilW, PilE, and PilX, but not the major pilin PilA, suggesting that different pilin forms may have distinct cellular functions. Together these results suggest that surface signaling may be an integrated system with feedbacks and cross-talk among multiple signaling pathways.

\section{Results}

QS master regulator LasR is upregulated on a surface. Multiple surface-associated behaviors of $P$. aeruginosa require $\mathrm{QS}^{3,28}$, including virulence induction and swarming motility ${ }^{29}$. QS and surface sensing pathways have been considered to function independently because las $R$ is not required for many of the transcriptional changes associated with surface-association. However, the significant overlap in QS and surface-dependent phenotypes suggests that these pathways may be interconnected. Consequently, we sought to determine how surface-association influences QS. We compared the mRNA abundance of the QS master regulator las $R$ in planktonic and surface-associated cells (Fig. 1a) by both RNA-seq (Fig. 1b) and qRT-PCR (Fig. 1c) in conditions that we previously showed are sufficient to induce virulence ${ }^{3}$. We found that lasR expression is upregulated $\sim 2$-fold after $1.5 \mathrm{~h}$ of attachment, as confirmed by both assays (Fig. 1b, c). In contrast, a well-characterized direct target of LasR, lasI, did not show the same surface induction in either assay (Fig. $1 \mathrm{~b}$ and Supplementary Fig. 1).

To determine if the increase in las $R$ levels upon surfaceassociation is a result of cells experiencing a higher effective concentration of 3OC12-HSL AI from being confined on a surface, we measured the surface-associated las $R$ induction in a lasI mutant, which cannot synthesize 3OC12-HSL. Expression of lasR still increased after surface-association in a lasI mutant (Fig. 1c), suggesting that the changes in las $R$ expression may be induced by surface-association itself. This hypothesis is further supported by the fact that most LasR-regulated QS genes are not upregulated upon surface-association (Supplementary Data 1), suggesting that surface-association does not merely reflect strong activation of planktonic QS signaling.

Surface-associated cells are more sensitive to QS. The presence of elevated lasR mRNA levels suggests that surface-associated cells might be hyper-responsive to 3OC12-HSL. To test this hypothesis, we examined the expression of three targets that are 3OC12-HSL/LasR-dependent but surface-independent, lasI, rhlR, and $p q s A$. We treated cells with $1 \mu \mathrm{M}$ exogenous 3OC12-HSL during the $1.5 \mathrm{~h}$ of surface attachment, during which lasR increases twofold. Upon 3OC12-HSL addition, lasI, rhlR, and pqsA mRNA levels increased significantly more in surfaceassociated cells than in planktonic cells (Fig. 2a-c). The surfaceassociated hyper-induction of 3OC12-HSL targets was completely dependent on the presence of LasR as it was eliminated in a lasR deletion strain (Fig. 2a).

As an additional measure of the sensitized QS response, we compared the dose-response curves of surface-attached and planktonic cells to 3OC12-HSL. Using a fluorescent reporter 
a
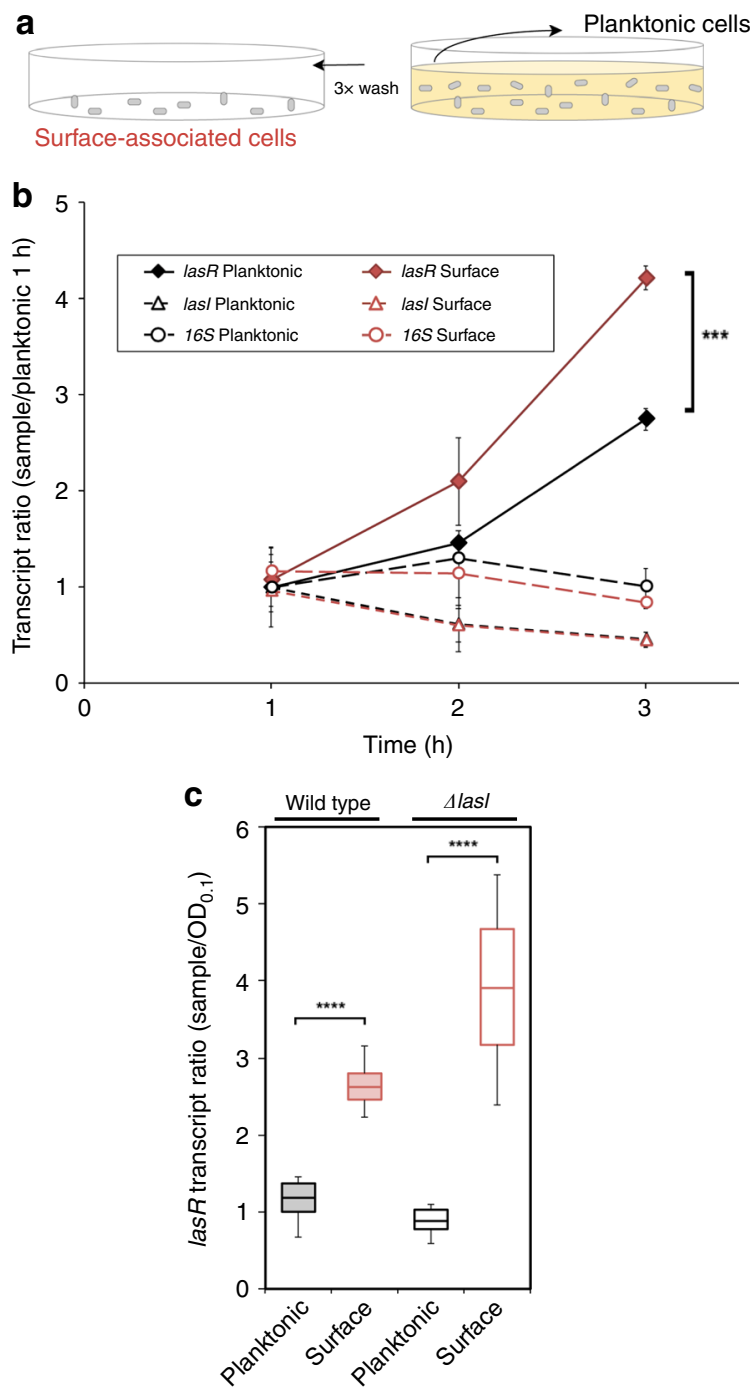

Fig. 1 Surface association increases the expression of the QS master regulator lasR. a Diagram of the experimental procedure for obtaining surface-attached and planktonic cells. b Expression of las $R$ and lasl and control $16 \mathrm{~S}$ from RNA-seq time course experiments normalized by the planktonic expression at $1 \mathrm{~h}(n=3)$. The biological replicates were averaged, with the standard deviation marked with error bars. In this and all subsequent plots planktonic conditions are shown in black and surfaceattached conditions are shown in red. c las $R$ response in a $\Delta$ lasl mutant that cannot produce 30C12-HSL from qRT-PCR at timepoint of $1.5 \mathrm{~h}(n=9)$. This and all subsequent qRT-PCR experiments are normalized by both $5 \mathrm{~S}$ rRNA and OD. Error bars in plots represent standard deviation, and boxes indicate the interquartile range with the center representing the mean. $A$ two tailed student's $t$-test was performed for comparison between samples, and significance is denoted by the asterisks $\left({ }^{\star} p<0.01,{ }^{\star \star} p<0.01,{ }^{\star \star \star} p<\right.$ $\left.0.001,{ }^{\star \star \star \star} p<0.0001\right)$. Source data are provided as a Source Data file

fusion to the lasI promoter in a strain that cannot produce its own 3OC12-HSL, we found that the response to 3OC12-HSL saturates at significantly higher levels when cells are surfaceattached (Supplementary Fig. 1A, B). These fluorescent reporter assays were performed using a glass surface, while the RNA-seq and $\mathrm{qRT}-\mathrm{PCR}$ assays were performed using polysterene surfaces, indicating that the sensitization of QS is not dependent on the composition of the surface. These results also indicate that at lower 3OC12-HSL concentrations that are more likely to be physiologically relevant, there is significantly higher induction of LasR targets when cells are surface-attached (Supplementary
Fig. 1A). Analysis of the individual cell responses indicate that the QS responses are roughly normally distributed, suggesting that surface attachment increases the overall sensitivity of the population rather than causing a small subpopulation to become extremely sensitive (Supplementary Fig. 1C). Our findings that planktonic cells do not strongly induce LasR targets by $1.5 \mathrm{~h}$ of 3OC12-HSL treatment and have lower target induction at saturation are consistent with a previous report that high levels of 3OC12-HSL are not sufficient to fully induce most LasR targets at low cell density ${ }^{18,30}$. Since mRNA levels do not always reflect protein levels, we generated a LasR-FLAG fusion to directly assess LasR protein levels by Western blot. This assay confirmed that LasR-FLAG protein increases more in surface-attached cells than in planktonic cells in the presence of 3OC12-HSL (Supplementary Fig. 2).

While the results above focus on LasR targets that are more strongly activated upon surface attachment, there are also targets that appear to only become activated by LasR in the surfaceassociated context. For example, previous studies on QS in planktonic cells found that 3OC12-HSL does not transcriptionally induce its LasR receptor ${ }^{15}$. Consistent with these reports, we found that 3OC12-HSL addition had little effect on lasR expression in planktonic cells (Fig. 2D). In contrast, 3OC12HSL induced lasR 8.5-fold more in surface-associated cells than in untreated planktonic cells (Fig. 2D). These lasR levels were 2 -fold greater than those of stationary-phase planktonic cells at higher density $\left(\mathrm{OD}_{600}\right.$ of 4.0 for the stationary phase cells as opposed to $\mathrm{OD}_{600}$ of 0.6 for the surface-associated cells) (Fig. 2e). These results reveal a condition-specific positive feedback loop in which 3OC12-HSL-bound LasR induces its own expression in surfaceassociated cells but not in planktonic cells.

The above results suggest that lasR induction may be an early response to surface-association. We note that while the extent of induction of LasR within $1.5 \mathrm{~h}$ of being introduced to a surface is modest, this induction represents a population-averaged lower limit that likely under-reports the extent of induction due to individual cells only associating with the surface for only a fraction of that timespan. For brevity, we henceforth refer to the increased QS sensitivity upon surface-association as surfaceprimed QS.

Surface-primed QS requires the sRNA Lrs1. Our findings indicate that surface-primed QS depends on lasR, but what factors promote lasR induction upon surface-association? sRNAs represent intriguing candidate regulators as they have been implicated in surface-associated behaviors, like biofilm formation and virulence, as well as in QS signaling pathways ${ }^{31-34}$. We thus examined surface-primed QS upon deletion of the RNA chaperone $\mathrm{Hfq}$, which is required for the stability and function of many sRNAs ${ }^{35}$. Addition of 3 OC12-HSL to a $\Delta h f q$ strain no longer showed induction of lasR mRNA upon surface-association (Fig. 3b).

Since the dependence of surface-primed QS on Hfq suggested the possible involvement of an sRNA, we performed RNA-seq in the conditions where cells become sensitized to QS and mapped our data set to a previously-compiled list of predicted sRNAs in $P$. aeruginosa PA14 ${ }^{21}$. We focused on one sRNA, Lrs1, that was significantly upregulated upon surface-association (Fig. 3c), showed similar qualitative expression trends to those of lasR (Fig. 1b), and was previously shown to be regulated by LasR ${ }^{21}$. Deletion of $l r s 1$ reduced lasR transcripts to undetectable levels even upon 3OC12-HSL addition to surface-associated cells (Fig. 3d). Furthermore, constitutive overexpression of Lrs1 caused lasR to become expressed in planktonic cells in a manner that did not significantly increase in surface-associated cells (Fig. 3d). 
a

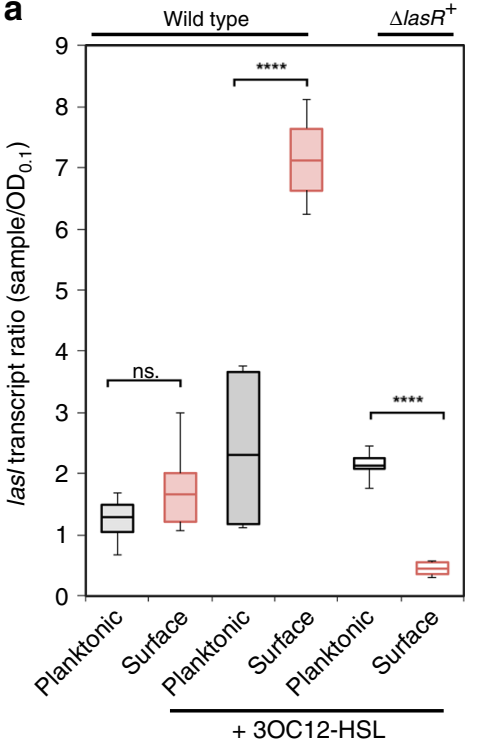

b

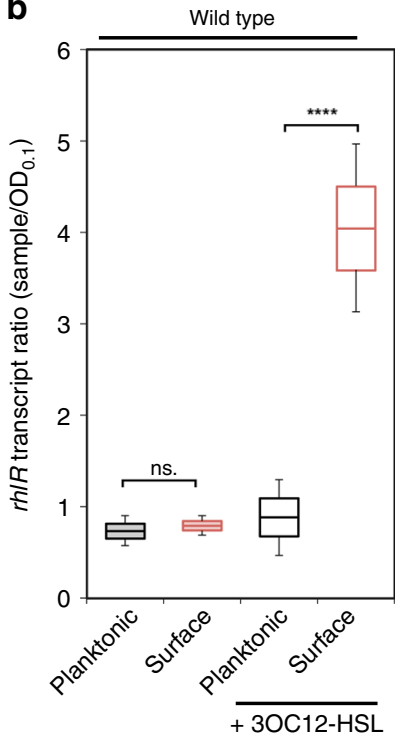

C
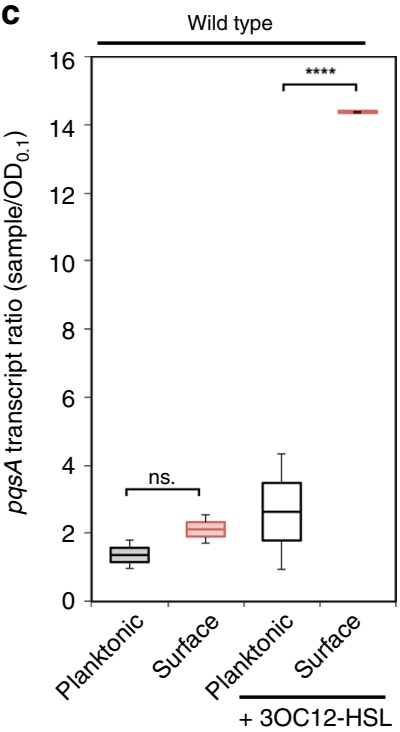
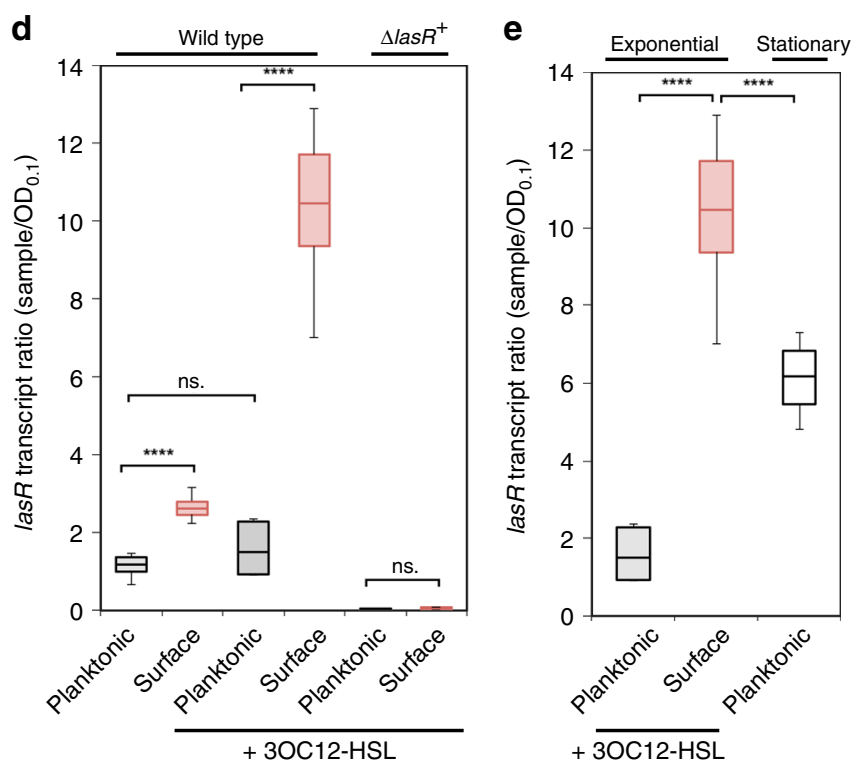

Fig. 2 Surface-association increases QS response. qRT-PCR levels normalized to OD and $5 \mathrm{~S}$ rRNA for las/ a, rhIR $\mathbf{b}$, pqsA $\mathbf{c}$, and las $R \mathbf{d}$ expression after $1.5 \mathrm{~h}$ treatment with $30 \mathrm{C} 12-\mathrm{HSL}$ or vehicle control. ${ }^{+}$( $\Delta$ las $R$ mutant data collected after $3 \mathrm{~h}$ treatment due to decreased surface-attachment phenotype).

e Expression of lasR in exponential phase compared to stationary phase by qRT-PCR. Error bars in plots represent standard deviation, and boxes indicate 25/75 data with the center representing the mean. A two tailed student's $t$-test was performed to determine significance between samples and is denoted with asterisks $(n=9)$ (ns. $=$ no significance, ${ }^{\star} p<0.01,{ }^{\star \star \star \star} p<0.0001$ ). Source data are provided as a Source Data file

These results suggest that constitutive Lrs1 expression induces lasR while disrupting its normal surface-sensitive regulation.

On the chromosome, $l r s 1$ is encoded upstream of the promoter region of an operon encoding genes of another QS pathway, the $p q s A-E$ genes that control PQS production (Fig. 3a). To differentiate whether the effects of deleting $l r s 1$ are due to the loss of Lrs1 itself or an indirect loss of $p q s A$ promoter activity, we examined the QS response in a $\triangle p q s A-E$ mutant. We found that unlike $\Delta l r s 1, \Delta p q s A-E$ maintained lasR induction by 3OC12-HSL in surface-associated cells (Fig. $3 \mathrm{e}$ ). We note that while $\Delta p q s A-E$ maintains surface-sensitive las $R$ induction, the absolute levels of lasR change in this background, consistent with previous suggestions of significant feedbacks and interconnectivity between the $P$. aeruginosa QS systems ${ }^{9}$. Combined with the fact that Lrs1 overexpression can induce lasR expression even when not expressed from its native locus, these data suggest that Lrs1 acts as a PQS-independent trans-acting regulator of lasR.
The requirement of Lrs1 for lasR induction upon surfaceassociation suggests that Lrs1 should also be required for the QS responses downstream of LasR. We thus examined the response of the LasR target, lasI, to 3OC12-HSL in planktonic and surfaceassociated cells. These results demonstrated that $\Delta l r s 1$ abolishes hyper-induction of lasI upon surface-association while $\Delta p q s A-E$ does not. In addition, constitutive Lrs1 overexpression induces lasI but renders it surface-independent (Supplementary Fig. 3). Finally, we assessed the effect of $\Delta l r s 1$ on another QS-dependent phenotype using an Elastin-Congo Red elastase activity. As predicted by the effects of Lrs1 on LasR, $\Delta l r s 1$ reduced elastase activities to levels comparable to those of $\Delta l a s R$, while constitutively overexpressing Lrs1 maintained elastase activity at or above WT levels (Fig. 3f).

A previous study demonstrated that LasR directly regulates the expression of $l r s 1^{21}$. Here we show that Lrs1 is also required for the expression of lasR (Fig. 3d). Thus, lrs 1 and lasR appear to be 
a

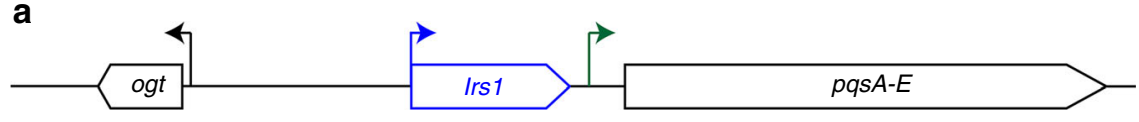

b

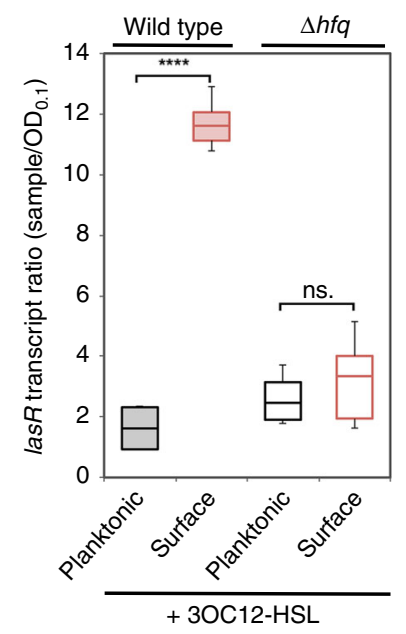

C

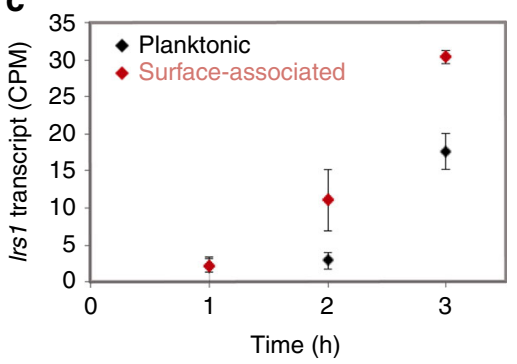

d

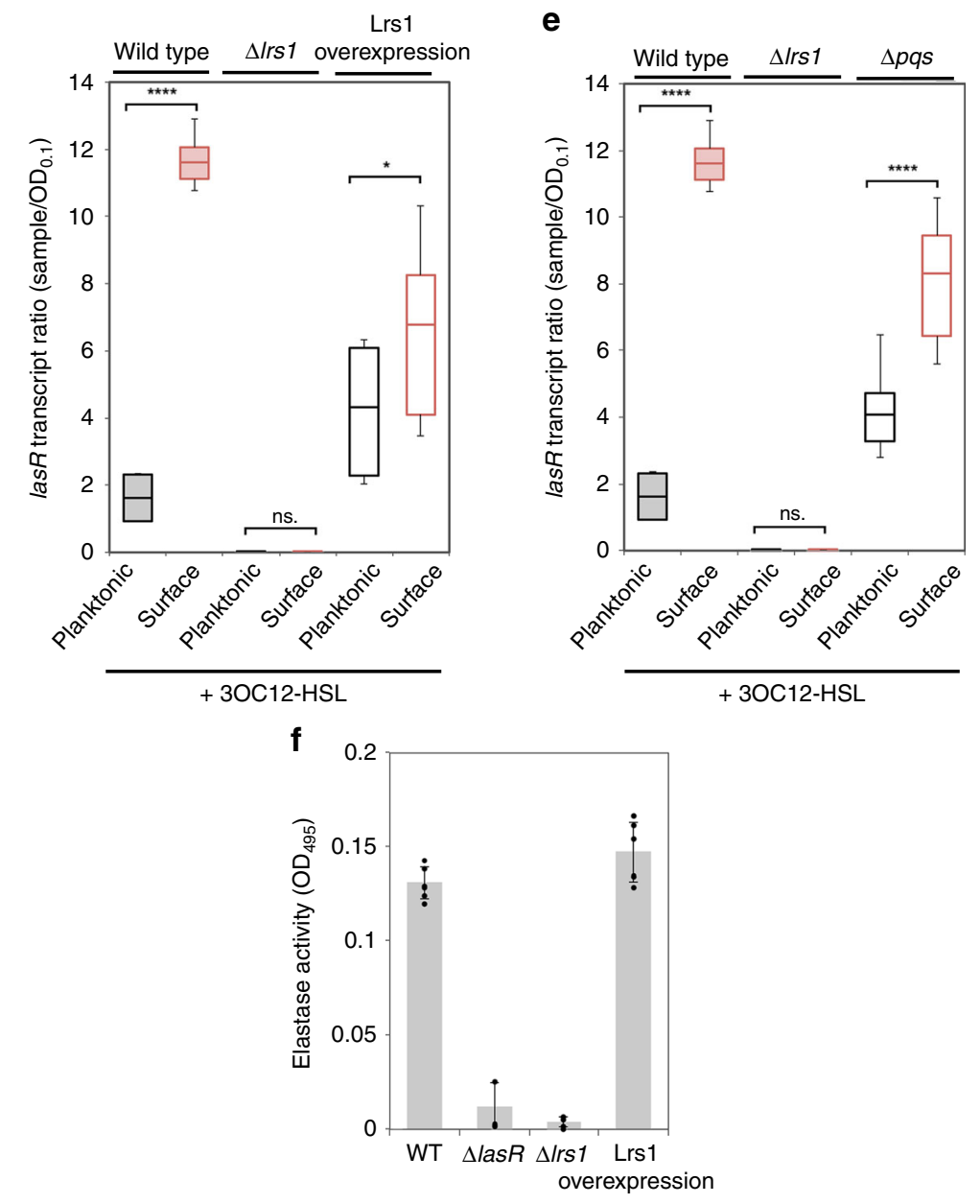

Fig. 3 sRNA Lrs1 is required for surface-primed QS. a Depiction of Irs1 location in the P. aeruginosa genome. b lasR transcript levels from qRT-PCR. c Lrs1 levels from RNA-seq data for planktonic and surface-attached cells. $\mathbf{d}$ las $R$ abundance by qRT-PCR in $\Delta / r s 1$ and upon Lrs1 overexpression. elasR abundance by qRT-PCR in $\triangle p q s A-E$. $\mathbf{f}$ Congo Red-Elastase assay comparing WT, $\Delta$ las $R, \Delta / r s 1$ and Lrs1 overexpression. Individual data represented by black points ( $n=$ 6). Error bars in plots represent standard deviation and boxes indicate $25 / 75$ data with the center representing the mean. A two-tailed student's $t$-test was performed to determine significance between samples and is denoted by asterisks $(n=9)$ (ns. = no significance, $\left.{ }^{\star} p<0.01,{ }^{\star \star \star \star} p<0.0001\right)$. Source data are provided as a Source Data file 

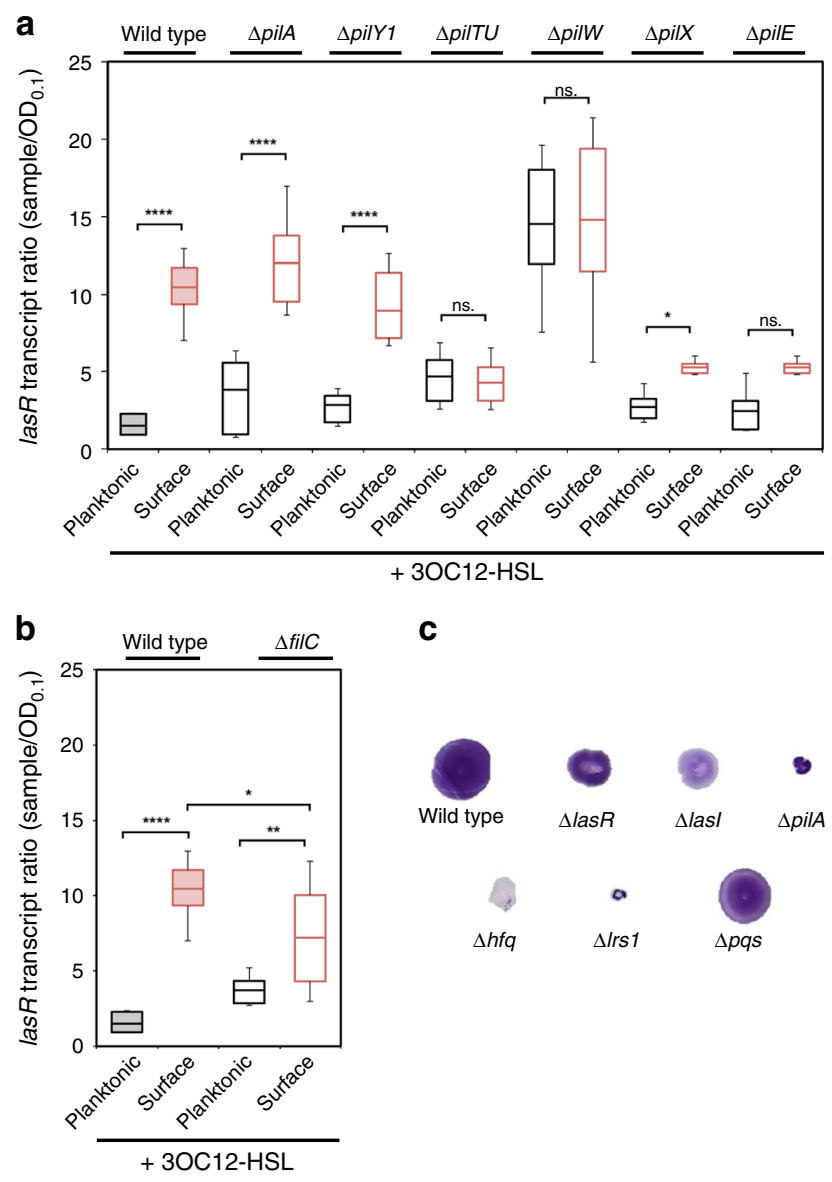

Fig. 4 Surface-primed QS is mediated through TFP retraction motors and minor pilins. a las $R$ abundance as measured by qRT-PCR in TFP mutants: $\triangle$ pilA, $\Delta$ pilY1, $\Delta$ pilTU, $\Delta$ pilW, $\Delta$ pilX, and $\Delta$ pilE. $\mathbf{b}$ las $R$ abundance as measured by qRT-PCR in flagella mutant $\Delta$ fliC. c TFP-dependent twitching phenotype in QS ( $\Delta$ las $R, \Delta$ lasl) and sRNA mutants $(\Delta h f q, \Delta / r s 1)$ with controls $\Delta p q s A-E$ and $\triangle$ pilA. Error bars in plots represent standard deviation and boxes indicate 25/75 data with the center representing the mean. A two-tailed student's $t$-test was performed to determine significance between samples and is denoted by asterisks $(n=9)$ (ns. $=$ no significance, ${ }^{*} p<0.01,{ }^{* *} p<0.01$, ${ }^{\star * \star *} p<0.0001$ ). Source data are provided as a Source Data file

interdependent, with each gene affecting the expression of the other. These data suggest a model in which upon surfaceassociation, the induction of Lrs1 induces LasR, which positively feedbacks to yet further induce $\operatorname{lrs} 1$, generating a robustly surfaceprimed QS response.

TFP retraction motors and minor pilins affect surface-primed QS. Several systems have recently been implicated as surface sensors in $P$. aeruginosa or other bacteria. These include surface structures such as flagella, TFP and PilY18,23,36. We thus tested mutants lacking these structures and their accessory factors for their involvement in surface-primed QS (Fig. 4). Surprisingly, we found that surface-primed QS was not strongly dependent on flagella (Fig. 4b), PilY1, or the major pilin PilA. However, surfaceprimed QS was influenced by the TFP retraction motors, PilT and PilU, and the minor pilins, PilE, PilX, and PilW (Fig. 4a). pilTU mutants appeared surface-blind in that they displayed low lasR levels regardless of whether they were planktonic or on a surface. Similarly, the pilE and pilX minor pilin mutants significantly reduced lasR induction upon surface attachment. In contrast,
pilW mutants appeared to be surface-locked in that they had high lasR levels in both planktonic and surface-associated conditions. Since pilA had no effect on las $R$ regulation, these results suggest that the minor pilins and PilTU motors have a PilA-independent role in surface signaling and that PilEX and PilW have opposing functions in this process.

Given the unexpected nature of the PilA-independent pilW dependence we confirmed that all of the mutants tested maintained their predicted twitching phenotypes (Supplementary Fig. 4). We also confirmed that the twitching defects of lasR and lasI can be complemented such that their defects are not due to compensatory mutations (Supplementary Fig. 5). To quantify twitching we used a plate twitching assay in which the diameter of the twitch zone serves as a measure of twitching as indicated by the small diameter of a twitching-defective pilA mutant control. We also examined twitching in $\Delta l r s 1$ mutants and found that $\Delta l r s 1$ disrupts twitching motility (Fig. 4c). This result indicates that the Lrs1-dependent surface-associated signaling pathway affects additional surface-associated behaviors beyond QS.

\section{Discussion}

LasR can function at the top of the QS cascade in $P$. aeruginosa, but relatively little is known about the regulation of LasR itself ${ }^{9}$. Here we show that lasR is upregulated upon bacterial surfaceassociation and that this upregulation is functional as it causes cells to respond more strongly to the LasR ligand, 3OC12-HSL. These results suggest that QS can operate in two different regimes in $P$. aeruginosa, with the same level of autoinducer synthesis causing low levels of QS activation in planktonic cells and high levels of QS activation in surface-associated cells. The fact that the system saturates at a low level in low-density planktonic cultures (higher autoinducer levels do not increase activation of planktonic cells) indicates that the QS activation achievable by planktonic cells is capped. These findings support a threshold model in which strong LasR targets that can be activated with lower LasR concentrations are induced in both planktonic and surface-associated cells, while weaker LasR targets that require higher LasR concentrations for induction are only induced in surface-associated cells.

In the future it will be interesting to determine if and how the coupled priming of surface sensing and QS benefits $P$. aeruginosa and whether similar coupling occurs in other systems. For example, the threshold for the number of community members needed to launch a successful behavior such as virulence induction could be lower if the bacteria are already on the surface of a host cell. Alternatively, the bacteria could sense one another as surfaces to hyper-activate community behaviors within aggregates like biofilms or anticipate the future accumulation of progeny upon colonizing an abiotic surface.

In this work, we observe that the induction of lasR upon surface association forms a positive feedback loop involving the sRNA Lrs1. It is interesting to note that sRNAs oftentimes hold crucial positions in regulatory circuits including QS, virulence and biofilm formation pathways in bacteria ranging from Vibrio cholerae to Streptococcus pneumoniae $34,37,38$. The versatility of sRNAs to act as both positive and negative regulators of gene expression, and their ability to simultaneously control multiple targets make sRNAs ideal central nodes within regulatory networks ${ }^{39,40}$. In addition, bacterial sRNAs differ from regulatory proteins such as transcription factors with regard to their regulation dynamics. These distinct characteristics may be relevant for the timely and precise adjustment of the gene expression profile in response to surface sensing.

Typically, bacterial sRNAs engage short, often imperfect basepairing interactions with target mRNAs to alter the stability and/ 
or translation of these transcripts ${ }^{41}$. Whether lasR mRNA is directly controlled by Lrs1 through an RNA-RNA interaction in response to surface attachment remains to be determined. It is also possible that Lrs1 affects lasR expression indirectly by modulating another factor that in turn regulates LasR. Expression of lasR increases in a growth-dependent manner, but basal transcription in the absence of auto-inducers reveals that las $R$ expression is not solely controlled by $\mathrm{QS}^{17}$. Several factors have been identified to adjust las $R$ transcription, including positive regulation via $\operatorname{Vfr}^{15}$ and the GacA/GacS system ${ }^{42}$, as well as negative regulation through $\mathrm{AlgR}^{43}$. It will be interesting to determine the target repertoire of Lrs1 in a future study, and to elucidate its role in las $R$ regulation.

Our findings suggest that LasR signaling is similar to that of many other QS systems in surface-associated cells. Meanwhile, the features of LasR signaling that were previously thought to be idiosyncratic (such as the lack of feedback onto lasR expression) could reflect the lack of the pathway's complete activation in planktonic cells. The surface-specific increase of lasR expression may also explain why so many surface phenotypes in P. aeruginosa are QS dependent.

The fundamental question of how bacteria sense the presence of a surface is also poorly understood. P. aeruginosa have emerged as a model system for surface sensing, with multiple distinct surface-sensing pathways. One pathway involves TFP and the Chp chemosensory system to induce cAMP and the Vfr transcription factor, which activates the expression of virulence factors ${ }^{7,8}$. Another pathway upregulates c-di-GMP through PilY1 and possible involvement of TFP, which influences biofilm formation ${ }^{7,8}$. In all previous cases where TFP have been implicated in $P$. aeruginosa surface sensing, the behavior requires the major pilin subunit, $\mathrm{PilA}^{7,8}$. However, our observations suggest that surface sensing mechanisms may be even more complex as we identify a surface sensing pathway that is independent of PilA yet influenced by the minor pilins PilW, PilE, and PilX, and the retraction motors PilTU. Minor pilins are generally thought to be integrated into the TFP fiber, and hypothesized to serve as adhesins to specific surfaces ${ }^{44}$. However, PilW may be distinct from other minor pilins as it does not bind to $\mathrm{FimU}^{45}$ and analysis of protein levels suggests that while other minor pilins cannot be secreted in the absence of PilA, a small amount of PilW can become extracellular even without the major pilin 45 .

In addition to being independent of PilA, the pilW response is surprising in that it appears "surface-locked", with surfaceprimed QS even in the planktonic state. This result suggests that PilW somehow inhibits lasR induction while the other minor pilins PilE and PilX stimulate lasR induction. There are at least two models for how $P$. aeruginosa might detect the surface in a manner that is independent of PilA, inhibited by PilW, and dependent on PilTU and PilEX. Pilin proteins often have feedback mechanisms to increase expression when the pili are active $^{46}$. It is thus possible that intracellular PilW inhibits las $R$ induction through Lrs1 in a manner that is antagonized by the function of PilTU and PilEX. Alternatively, P. aeruginosa may respond to mechanical tension in surface structures, for example by PilTU causing retraction of fibers based on PilEX that are inhibited by PilW. However, for this model to be viable the minor pilins would have to form extracellular structures independently of PilA, which has not been previously reported. In any event, our data suggest that the mechanical and signaling roles of the minor pilin subunits are worthy of further investigation in future studies as they may play important roles independently of the major pilin subunit.

Regardless of the mechanistic details, our findings that surfaceassociated cells are more sensitive to QS compared to their planktonic counterparts have implications for how $P$. aeruginosa coordinates the multiple behaviors in exhibits on surfaces such as biofilm formation, virulence induction, and surface motility. Previous studies demonstrated that QS is required for other surface behaviors, but transcriptional profiling suggested that this relationship can be independent ${ }^{3}$. Our current work provides a potential explanation for this apparent paradox. We suggest that by using an independent surface sensing pathway to sensitize themselves to QS, $P$. aeruginosa cells can effectively differentiate, allowing them to use the same QS cues already available to access states they could not access in the absence of a surface. In this sense our current work supports the previous suggestion of surface sensing and cell density forming an "AND gate"-like regulatory mechanism where $P$. aeruginosa only commits to energetically costly behaviors such as virulence induction if it is present in conditions where it is both on a surface sufficiently rigid to trigger surface sensing, and at high enough densities to trigger $\mathrm{QS}^{3}$. We thus effectively expand the canonical QS pathway for surface-associated cells; cells detect a surface and then prime themselves for a QS response that is only fully activated once the cells reach high cell density. While surface sensing and QS responses are coupled, they are still independent because surfaceassociated responses cannot be recreated by simply increasing the density of cells and QS cannot be fully activated in the absence of surface-association. Further elucidating these signaling cascades may thus respresent new ways of blocking virulence induction and biofilm formation for the development of novel anti-infective therapies.

\section{Method}

Bacterial strains and growth conditions. P. aeruginosa strain PA14 is referred to as wild type (WT) throughout this study. All strains used in this study are listed in Table 1, all oligonucleotides are summarized in Table 2. Bacteria were routinely grown in Luria broth (LB) or LB solidified with $1.5 \%$ agar. For qRT-PCR and RNAseq experiments, overnight cultures were diluted $1: 1000$ in $\mathrm{LB}$ and grown at $37^{\circ} \mathrm{C}$ to $\mathrm{OD}_{600} 0.1$. Cultures were transferred to petri dishes, and incubated on an orbital shaker at $100 \mathrm{rpm}$ and $37^{\circ} \mathrm{C}$. For the planktonic conditions, aliquots of culture

\section{Table 1 Bacterial strains}

\begin{tabular}{|c|c|c|}
\hline Strain Name & Description & Reference \\
\hline Wild type (WT) & $\begin{array}{l}\text { P. aeruginosa strain PA14, clinical } \\
\text { isolate from burn wound }\end{array}$ & 53 \\
\hline$\Delta$ lasR & PA14 AlasR & 28 \\
\hline$\Delta$ lasl & PA14 $\Delta$ lasl & 54 \\
\hline$\Delta h f q$ & PA14 $\Delta h f q$ & 21 \\
\hline$\Delta / r s 1$ & PA14 Alrs1 & 21 \\
\hline Lrs1 overexpression & PA14 glmS::[P $P_{\left.\mathrm{Al} / 04 / 03^{-}-\operatorname{rs} 1\right]}$ & This work \\
\hline$\Delta p q s A-E$ & PA14 $\triangle p q s A B C D E$ & This work \\
\hline$\Delta$ fliC & $\begin{array}{l}\text { PA14 P Pro::yfp attB::[P }\left[P_{A 1 / 04 / 03^{-}}\right. \\
m \text { Cherry } \Delta \text { fliC }\end{array}$ & \\
\hline$\Delta p i l A$ & $\begin{array}{l}\text { PA14 Pfro::yfp attB::[ }\left[P_{A 1 / 04 / 03^{-}}\right. \\
m C h e r r y] \Delta \text { pilA }\end{array}$ & 47 \\
\hline$\Delta$ pilC & PA14 ApilC & 22 \\
\hline$\Delta$ pilD & PA14 $\Delta$ pilD & 55 \\
\hline$\Delta p i l E$ & PA14 $\Delta$ pilE & 55 \\
\hline$\Delta$ pilTU & 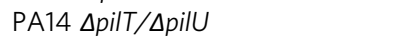 & 56 \\
\hline$\Delta p i l W$ & PA14 $\Delta$ pilW & 55 \\
\hline$\Delta p i l X$ & PA14 $\Delta$ pilX & 55 \\
\hline$\Delta$ pilY1 & PA14 $\Delta$ pilY1 & 57 \\
\hline FLAG-lasR & PA14 FLAG-IasR & This work \\
\hline$\Delta$ lasR lasRl::attB & PA14 DlasR lasRl::attB & This work \\
\hline$\Delta$ lasl lasRl::attB & PA14 Dlasl lasRl::attB & This work \\
\hline$P_{\text {lasl }}-m$ Cherry & PA14 $P_{\text {lasl-mCherry:::attB }}$ & This work \\
\hline$\Delta$ lasl $P_{\text {lasl-mCherry }}$ & PA14 $\Delta$ lasl $P_{\text {lasl }}-m$ Cherry::attB & This work \\
\hline $\begin{array}{l}\text { FLAG-lasR } P_{\text {lasl }}{ }^{-} \\
\text {mCherry::attB }\end{array}$ & PA14 FLAG-lasR P Plas/-mCherry::attB & This work \\
\hline S17 & E. coli & \\
\hline
\end{tabular}


Table 2 Oligonucleotides

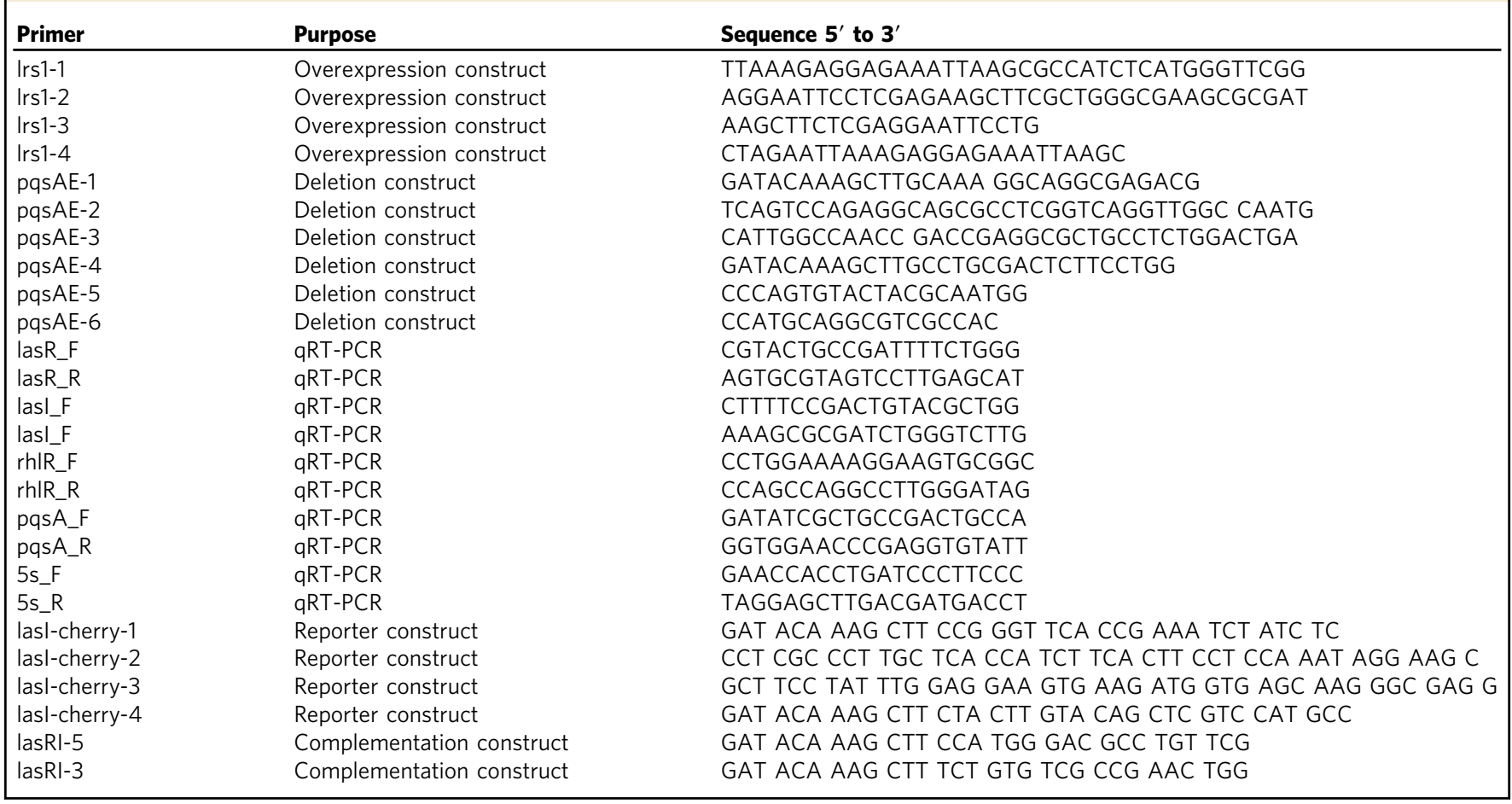

media were centrifuged, and the cell pellets were resuspended in lysis solution (TE buffer $\mathrm{pH} 8.0,0.5 \mathrm{mg} / \mathrm{mL}$ lysozyme (Sigma), $1 \% \mathrm{SDS}$ ). For the surface attached condition, culture media was removed from the petri dish, and surface-attached cells were washed three times with DB $\left(5 \mathrm{mM} \mathrm{KH}_{2} \mathrm{PO}_{4}, 5 \mathrm{mM} \mathrm{Na}_{2} \mathrm{HPO}_{4}, 2 \mathrm{mM}\right.$ $\mathrm{MgCl}_{2}, 1 \mathrm{mM} \mathrm{CaCl}$, at $\mathrm{pH}$ 6.5). Lysis solution was added directly to the petri dish, and the sample was collected from the dish using a sterile cell scraper. For the RNA-Seq experiment, samples were collected at time points 1,2 , and $3 \mathrm{~h}$. For qRTPCR experiments, samples were collected at $1.5 \mathrm{~h}$. 30C12-HSL (Sigma) dissolved in DMSO was added to a final concentration of $1 \mu \mathrm{M}$, unless otherwise stated.

Strain construction. To generate an Lrs1 overexpression strain, the $\operatorname{lr} 1$ gene was amplified by PCR (primer pair lrs1-1, lrs1-2) and inserted into the pUC18-miniTn7 derivative, $\mathrm{pAS}^{477}$ (amplified by PCR using primer pair lrs1-3, lrs1-4), using Gibson Assembly ${ }^{48}$. The resulting construct was integrated into the T7 phage attachment site of wild-type PA14 following the procedures of Choi et al. ${ }^{49}$. An unmarked in-frame deletion of the pqsABCDE operon was generated using twostep allelic exchange as described before ${ }^{50}$. The regions 500-bp upstream and downstream of the operon were amplified by PCR (pqsAE-1, pqsAE-2) and (pqsAE-3, pqsAE-4), respectively, fused through overlap extension PCR (SOEPCR), and ligated into the HindIII site of plasmid pEXG2. Deletions were confirmed using by colony PCR (using primer pair pqsAE-5, pqsAE-6). To generate the FLAG-tagged lasR strain, a roughly 800-bp sequence containing 3xFLAG motif fused to the N-terminus lasR gene was synthesized commercially. This fragment was cloned into the HindIII site of pEXG2, and FLAG-lasR was integrated onto the chromosome through allelic exchange. Proper insertion was confirmed by sequencing. To generate the lasRI complementation strain, the lasRI genes were amplified from gDNA using primer pair (lasRI-5, lasRI-3) and cloned into the HindIII site of the mini-CTX plasmid. The CTX-2 construct was integrated into the chromosome of lasR and lasI mutants at the attB phage attachment site. To generate the $\mathrm{P}_{\text {lasl }}$ mCherry promoter fusion, a $\sim 300 \mathrm{bp}$ fragment upstream of the lasI gene was amplified from gDNA using primer pair (lasI-cherry-1, lasI-cherry2 ). This fragment was fused by SOE-PCR to a promoterless $m$ Cherry fragment, amplified from plasmid pAS08 using primer pair (lasI-cherry-3, lasI-cherry-4). The resulting fusion was cloned into mini-CTX-2 and integrated onto the chromosome at the $a t t B$ site.

RNA isolation. Total RNA was from isolated from surface-attached and planktonic cells as previously described ${ }^{3}$. In brief, planktonic cells were collected by pelleting $600 \mu \mathrm{L}$ of the liquid culture in the dish. The supernatant was removed and immediately lysed with lysis solution (TE buffer $\mathrm{pH} 8.0,0.5 \mathrm{mg} / \mathrm{mL}$ lysozyme (Sigma), $1 \%$ SDS). Surface-associated cells were isolated by washing the dish three times with $\mathrm{DB}\left(5 \mathrm{mM} \mathrm{KH}_{2} \mathrm{PO}_{4}, 5 \mathrm{mM} \mathrm{Na}_{2} \mathrm{HPO}_{4}, 2 \mathrm{mM} \mathrm{MgCl}_{2}, 1 \mathrm{mM} \mathrm{CaCl}\right.$, at pH 6.5). Lysis solution was added to isolate surface-attached cells from the petri dish, and the sample was collected from the dish using a sterile cell scraper within a few minutes of the experimental time point. Total RNA was isolated from all samples collected in lysis solution using hot phenol extraction. Genomic DNA was removed from all total RNA samples by DNase I digestion.

qRT-PCR prep and analysis. For each experimental condition, cDNA was synthesized from $1.5 \mu \mathrm{g}$ total RNA using Superscript III (Invitrogen) following the manufacturer's recommendations. cDNA was mixed with gene-specific primer sets (Tables 2) and $2 \times$ PerfeCTa SYBR Green FastMix Low Rox (Quanta Bio), and run on a Viia7 384-Well Fast Real Time PCR System (Thermo Fisher) in the Princeton Genomics Core Facility. For each of the three biological replicates collected per sample, four technical replicates were run. CT values were converted to fold-change through the Pfaffl Method ${ }^{51} .5 \mathrm{~S}$ rRNA was used as an internal control, and the control condition present on each plate was RNA prepared from planktonic wildtype cells at $\mathrm{OD}_{600}$ of 0.1 . Each biological replicate was then averaged, and the average and standard deviation of the three biological replicates was plotted.

RNASeq library prep and analysis. Libraries for Illumina Sequencing of cDNA were constructed by the Princeton Genomics Core Facility. The integrity of total RNA samples (three biological replicates) was assessed on a Bioanalyzer 2100 using RNA 6000 Pico chip (Agilent Technologies, CA). For each sample, $0.5 \mu \mathrm{g}$ of RNA were treated with the Ribo-Zero Bacteria rRNA Removal Kit (Illumina, CA) to deplete the majority of ribosomal RNAs. The RNA was fragmented and converted to cDNA libraries using the PrepX RNA-seq library kit (Takara Bio USA) on the automated Apollo $324^{\mathrm{TM}}$ NGS Library Prep System (Clontech, CA). Libraryspecific barcodes were introduced to allow for multiplex sequencing. The RNA-seq libraries were examined on Agilent Bioanalyzer DNA High Sensitivity chips for size distribution, quantified using a Qubit fluorometer (Invitrogen, CA), and pooled at equal molar amounts. The library pools were denatured and sequenced on Illumina HiSeq 2500 Rapid flowcells as single-end $75 \mathrm{nt}$ reads according to the manufacturer's protocol. Raw sequencing reads were filtered by the Illumina HiSec Control Software, only the Pass-Filter (PF) reads were de-multiplexed allowing one mismatch, and used for further analysis.

Sequence files from the facility were processed with customized Python scripts from our lab (which are available on request), and were aligned to the $P$. aeruginosa UCBPP-PA14 genome using Bowtie $2^{52}$. Raw counts were divided by total number of reads per sample and converted to counts per million (CPM) for comparison. CPM for each biological replicate were averaged, and the standard deviation is indicated by error bars. Data in plots were normalized by the planktonic counts at $1 \mathrm{~h}$ for each gene for comparison.

Western blot analysis. For western blot analysis, cultures were grown in PS:DB media, which consists of DB and $10 \%(\mathrm{v} / \mathrm{v})$ PS medium $\left(10 \mathrm{~g} \mathrm{~L}^{-1}\right.$ Special Peptone (Oxoid), $7 \mathrm{~g} \mathrm{~L}^{-1}$ Yeast Extract (Oxoid), $10 \mathrm{mM} \mathrm{KH}_{2} \mathrm{PO}_{4}, 0.45 \mathrm{M} \mathrm{Na}_{2} \mathrm{HPO}_{4}, 15 \mathrm{~g} \mathrm{~L}^{-1}$ glucose, $20 \mathrm{nM}$ vitamin $\mathrm{B} 12,180 \mathrm{nM}$ Folic Acid, $\mathrm{pH}$ 6.5). Overnight cultures of 
FLAG-pqsA were diluted 1:100 in PS:DB and grown to $\mathrm{OD}_{600 \mathrm{~nm}}=0.5-0.6$ at $37^{\circ} \mathrm{C}$ with shaking $(250 \mathrm{rpm})$. Cultures were transferred to $22 \times 22 \mathrm{~cm}$ culture dishes and incubated for $1 \mathrm{~h}$ at $37^{\circ} \mathrm{C}$ on an orbital shaker $(80 \mathrm{rpm})$. Planktonic samples were isolated by aspirating the culture media. Surface-attached cells were washed twice in PBS and removed from the surface using a cell scraper. Cells were resuspended in loading buffer to equal concentrations, based on $\mathrm{OD}_{600 \mathrm{~nm}}$, and seperated on a $10 \%$ SDS-PAGE. Blots were probed using DYKDDDDK Tag (D6W5B) Rabbit monoclonal antibody (14793 S, Cell Signaling Technologies, Danvers, MA) at a 1:1000 dilution and a polyclonal antibody targeting the $\sigma^{70}$ subunit of RNA polymerase, provided by the Bassler lab, at 1:5000 dilution. Primary antibody was detected with anti-rabbit IgG, HRP-linked secondary antibody (7074 S, Cell Signaling Technologies, Danvers, MA) at 1:3000 dilution.

Analysis of fluorescent promoter fusions. Overnight cultures of $\Delta$ lasI expressing $P_{\text {las } I^{-}} m$ Cherry::attB or $P_{p q s A^{-}} m$ Cherry::attB fluorescent fusions were diluted 1:100 in PS:DB and incubated at $37^{\circ} \mathrm{C}$ with shaking $(250 \mathrm{rpm})$. Various amounts of $3 \mathrm{OC} 12$ HSL was added when cultures reached $\mathrm{OD}_{600 \mathrm{~nm}}=0.2$. When cultures reached $\mathrm{OD}_{600 \mathrm{~nm}}=0.5-0.6$, cultures were transferred to glass-bottom petri dishes (Mattek Corporation, Ashland, MA) and incubated for $1 \mathrm{~h}$ at $37^{\circ} \mathrm{C}$ on an orbital shaker $(80$ $\mathrm{rpm}$ ). Planktonic cells were isolated by aspirating the culture media. Surfaceattached cells were washed twice in PBS. Samples were covered with $1 \%$ agar pad and imaged using fluorescent microscopy.

Twitching assays. A toothpick was used to transfer cells from a solid plate to a $1.5 \% \mathrm{LB}$ agar pad. Plates were incubated in a humid box at $30^{\circ} \mathrm{C}$ for $48 \mathrm{~h}$. Twitching diameters were visualized by either incubating plates for $30 \mathrm{~min}$ in developer solution ( $50 \%$ methanol, $10 \%$ glacial acetic acid in $\mathrm{H}_{2} \mathrm{O}$ ), or by removing agar pads and incubating plates with $1 \%$ crystal violet in water for $10 \mathrm{~min}$. Plates were washed with water prior to imaging.

Congo red elastase assay. Cells were grown at $37^{\circ} \mathrm{C}$ overnight in $\mathrm{LB}$ and collected by centrifugation at $16,000 \times g$ for $1 \mathrm{~min}$. Supernatant were passed through a $0.22 \mu \mathrm{m}$ filter, and $100 \mu \mathrm{L}$ of supernatant was mixed with $900 \mu \mathrm{L}$ of $10 \mathrm{mM}$ $\mathrm{Na}_{2} \mathrm{HPO}_{4}$ and $10 \mathrm{mg}$ elastin-Congo red substrate (Sigma-Aldrich). After incubation for $2 \mathrm{~h}$ at $37^{\circ} \mathrm{C}$, and the samples were centrifuged at $16,000 \times g$ for $10 \mathrm{~min}$. The optical density of the supernatant was measured at $\mathrm{OD}_{495}$. Each biological sample had three technical replicates.

Reporting summary. Further information on research design is available in the Nature Research Reporting Summary linked to this article.

\section{Data avaliability}

The RNA sequencing data have been deposited in the SRA database at NCBI with accession code PRJNA528963.

\section{Code availability}

Customized Python scripts used to process RNA sequencing data are available on request.

Received: 20 December 2018 Accepted: 19 August 2019

Published online: 11 September 2019

\section{References}

1. Miller, M. B. \& Bassler, B. L. Quorum sensing in bacteria. Annu. Rev Microbiol. 55, 165-199 (2001).

2. O’Toole, G., Kaplan, H. B. \& Kolter, R. Biofilm formation as microbial development. Annu. Rev. Microbiol. 54, 49-79 (2000).

3. Siryaporn, A. et al. Surface attachment induces Pseudomonas aeruginosa virulence. PNAS 111, 16860-16865 (2014).

4. Persat, A., Inclan, Y. F., Engel, J. N., Stone, H. A. \& Gitai, Z. Type IV pili mechanochemically regulate virulence factors in Pseudomonas aeruginosa. Proc. Natl Acad. Sci. 112, 7563-7568 (2015).

5. Kearns, D. B. A field guide to bacterial swarming motility. Nat. Rev. Microbiol. 8, 634-644 (2010).

6. Mattick, J. S. Type IV pili and twitching motility. Annu. Rev. Microbiol. 56, 289-314 (2002).

7. Rodesney, C. A. et al. Mechanosensing of shear by Pseudomonas aeruginosa leads to increased levels of the cyclic-di-GMP signal initiating biofilm development. Proc. Natl Acad. Sci. 114, 5906-5911 (2017).

8. Luo, Y. et al. A Hierarchical Cascade Second Messengers Regul. Pseudomonas aeruginosa Surf. Behaviors_SUPP. 6, 1-11 (2015).

9. Lee, J. \& Zhang, L. The hierarchy quorum sensing network in Pseudomonas aeruginosa. Protein Cell 6, 26-41 (2014).
10. Seed, P. C., Passador, L. \& Iglewski, B. H. Activation of the Pseudomonas aeruginosa lasI gene by LasR and the Pseudomonas autoinducer PAI: an autoinduction regulatory hierarchy. J. Bacteriol. 177, 654-659 (1995).

11. Papenfort, K. \& Bassler, B. L. Quorum sensing signal-response systems in Gram-negative bacteria. Nat. Rev. Microbiol. 14, 576-588 (2016).

12. Schuster, M. \& Greenberg, E. P. Early activation of quorum sensing in Pseudomonas aeruginosa reveals the architecture of a complex regulon. BMC Genom. 8, 1-11 (2007).

13. Wagner, V. E., Bushnell, D., Passador, L., Brooks, A. I. \& Iglewski, B. H. Microarray analysis of Pseudomonas aeruginosa Quorum-sensing regulons: effects of growth phase and environment. J. Bacteriol. 185, 2080-2095 (2003).

14. Schuster, M., Lostroh, C. P., Ogi, T. \& Greenberg, E. P. Identifcation, timing, and signal specifcity of Pseudomonas aeruginosa quorum-controlled genes: a transcriptome analysis. J. Bacteriol. 185, 2066-2079 (2003).

15. Albus, A. M., Pesci, E. C., Runyen-janecky, L. J., West, S. E. H. \& Iglewski, B. $\mathrm{H}$. Vfr controls quorum sensing in Pseudomonas Vfr Controls Quorum Sensing in Pseudomonas aeruginosa. J. Bacteriol. 179, 3928-3935 (1997).

16. Siehnel, R. et al. A unique regulator controls the activation threshold of quorum-regulated genes in Pseudomonas aeruginosa. Proc. Natl Acad. Sci. 107, 7916-7921 (2010).

17. Pesci, E. C., Pearson, J. P., Seed, P. C. \& Iglewski, B. H. Regulation of las and rhl quorum sensing in Pseudomonas aeruginosa. J. Bacteriol. 179, 3127-3132 (1997).

18. Whiteley, M., Lee, K. M. \& Greenberg, E. P. Identification of genes controlled by quorum sensing in Pseudomonas aeruginosa. Proc. Natl Acad. Sci. USA 96, 13904-13909 (1999).

19. Winzer, K. et al. The Pseudomonas aeruginosa lectins PA-IL and. J. Bacteriol. 182, 6401-6411 (2000).

20. Papenfort, K. \& Vogel, J. Regulatory RNA in bacterial pathogens. Cell Host Microbe 8, 116-127 (2010).

21. Wurtzel, O., et al The single-nucleotide resolution transcriptome of Pseudomonas aeruginosa grown in body temperature. PLoS Pathog. 8, https:// doi.org/10.1371/journal.ppat.1002945 (2012)

22. O’Toole, G. A. \& Kolter, R. Flagellar and twitching motility are necessary for Pseudomonas aeruginosa biofilm development. Mol. Microbiol. 30, 295-304 (1998).

23. Hug, I., Deshpande, S., Sprecher, K. S., Pfohl, T. \& Jenal, U. Second messenger-mediated tactile response by a bacterial rotary motor. Science 358 531-534 (2017).

24. Cowles, K. N. \& Gitai, Z. Surface association and the MreB cytoskeleton regulate pilus production, localization and function in Pseudomonas aeruginosa. Mol. Microbiol. 76, 1411-1426 (2010).

25. Ellison, C. K. et al. Obstruction of pilus retraction stimulates bacterial surface sensing. Science 358, 535-538 (2017).

26. Hahn, H. P. The type- 4 pilus is the major virulence-associated adhesin of Pseudomonas aeruginosa-a review. Gene 192, 99-108 (1997).

27. Burrows, L. L. Twitching motility: type IV Pili in action. Annu. Rev. Microbiol. 66, 493-520 (2012).

28. O'Loughlin, C. T. et al. A quorum-sensing inhibitor blocks Pseudomonas aeruginosa virulence and biofilm formation. Proc. Natl Acad. Sci. 110, 17981-17986 (2013).

29. Copeland, M. F. \& Weibel, D. B. Bacterial swarming: a model system for studying dynamic self-assembly. Soft Matter 5, 1174-1187 (2009).

30. Diggle, S. P., Winzer, K., Lazdunski, A., Williams, P. \& Cámara, M. Advancing the quorum in Pseudomonas aeruginosa: MvaT and the regulation of $\mathrm{N}$ acylhomoserine lactone production and virulence gene expression. J. Bacteriol. 184, 2576-2586 (2002).

31. Bak, G. et al. Identification of novel sRNAs involved in biofilm formation, motility, and fimbriae formation in Escherichia coli. Sci. Rep. 5, https://doi. org/10.1038/srep15287 (2015).

32. Bardill, J. P. \& Hammer, B. K. Non-coding sRNAs regulate virulence in the bacterial pathogen Vibrio cholerae. RNA Biol. 9, 392-401 (2012).

33. Sonnleitner, E., Romeo, A. \& Bläsi, U. Small regulatory RNAs in Pseudomonas aeruginosa. RNA Biol. 9, 364-371 (2012).

34. Lenz, D. H. et al. The small RNA chaperone Hfq and multiple small RNAs control quorum sensing in Vibrio harveyi and Vibrio cholerae. Cell 118, 69-82 (2004).

35. Vogel, J. \& Luisi, B. F. Hfq and its constellation of RNA. Nat. Rev. Microbiol. 9, 578-589 (2011)

36. O’Toole, G. A. \& Wong, G. C. L. Sensational biofilms: surface sensing in bacteria. Curr. Opin. Microbiol. 30, 139-146 (2016).

37. Gripenland, J. et al. RNAs: regulators of bacterial virulence. Nat. Rev. Microbiol. 8, 857-866 (2010).

38. Mann, B. et al. Control of virulence by small RNAs in Streptococcus pneumoniae. PLoS Pathog. 8, 34 (2012).

39. Beisel, C. L. \& Storz, G. Base pairing small RNAs and their roles in globa regulatory networks. FEMS Microbiol. Rev. https://doi.org/10.1111/j.15746976.2010.00241.x (2010). 
40. Nitzan, M., Rehani, R. \& Margalit, H. Integration of bacterial small RNAs in regulatory networks. Annu. Rev. Biophys. https://doi.org/10.1146/annurevbiophys-070816-034058 (2017).

41. Waters, L. S. \& Storz, G. Regulatory RNAs in bacteria. Cell 136, 615-628 (2009).

42. Reimmann, C. et al. The global activator GacA of Pseudomonas aeruginosa PAO positively controls the production of the autoinducer N-butyrylhomoserine lactone and the formation of the virulence factors pyocyanin, cyanide, and lipase. Mol. Microbiol. 24, 309-319 (1997).

43. Ledgham, F., Soscia, C., Chakrabarty, A., Lazdunski, A. \& Foglino, M. Global regulation in Pseudomonas aeruginosa: The regulatory protein $\mathrm{AlgR} 2(\mathrm{AlgQ})$ acts as a modulator of quorum sensing. Res. Microbiol. 154, 207-213 (2003).

44. Helaine, S., Dyer, D. H., Nassif, X., Pelicic, V. \& Forest, K. T. 3D structure/ function analysis of PilX reveals how minor pilins can modulate the virulence properties of type IV pili. Proc. Natl Acad. Sci. 104, 15888-15893 (2007).

45. Nguyen, Y. et al. Pseudomonas aeruginosa minor pilins prime type IVa pilus assembly and promote surface display of the PilY1 adhesin. J. Biol. Chem. 290, 601-611 (2015).

46. Kilmury, S. L. N. \& Burrows, L. L. Type IV pilins regulate their own expression via direct intramembrane interactions with the sensor kinase Pils. Proc. Natl Acad. Sci. 113, 6017-6022 (2016).

47. Lorestani, A. Mechanical host-pathogen interactions of healthcare-associated infections. (2017).

48. Gibson, D. G. et al. Enzymatic assembly of DNA molecules up to several hundred kilobases. Nat. Methods 6, 343-345 (2009).

49. Choi, K.-H. \& Schweizer, H. P. mini-Tn7 insertion in bacteria with single attTn7 sites: example Pseudomonas aeruginosa. Nat. Protoc. 1, 153-161 (2006).

50. Hmelo, L. R. et al. Precision-engineering the Pseudomonas aeruginosa genome with two-step allelic exchange. Nat. Protoc. 10, 1820-1841 (2015).

51. Pfaffl, M. W. A new mathematical model for relative quantification in realtime RT-PCR. Nucleic Acids Res. 29, https://doi.org/10.1093/nar/29.9.e45 (2002).

52. Langmead, B. \& Salzberg, S. L. Fast gapped-read alignment with Bowtie 2. Nat. Methods https://doi.org/10.1038/nmeth.1923 (2012).

53. Rahme, L. et al. Common virulence factors for bacterial pathogenicity in plants and animals. Science 268, 1899-1902 (1995)

54. Mukherjee, S., Moustafa, D., Smith, C.D., Goldberg, J.B. \& Bassler, B.L. The RhlR quorum-sensing receptor controls Pseudomonas aeruginosa pathogenesis and biofilm development independently of its canonical homoserine lactone autoinducer. PLoS Pathog. 13, https://doi.org/10.1371/ journal.ppat.1006504 (2017).

55. Kuchma, S. L., Griffin, E. F. \& O'Toole, G. A. Minor pilins of the type IV pilus system participate in the negative regulation of swarming motility. J. Bacteriol. 194, 5388-5403 (2012).

56. Shen, Y., Siryaporn, A., Lecuyer, S., Gitai, Z. \& Stone, Ha Flow directs surfaceattached bacteria to twitch upstream. Biophys. J. 103, 146-151 (2012).
57. Kuchma, S. L. et al. Cyclic-di-GMP-mediated repression of swarming motility by Pseudomonas aeruginosa: the pilY1 gene and its impact on surfaceassociated behaviors. J. Bacteriol. 192, 2950-2964 (2010).

\section{Acknowledgements}

We wish to thank members of the Gitai and Shaevitz labs for helpful discussions and comments on the paper, the Bassler and Lory labs for strains and reagents, and Albert Siryaporn for technical assistance. Z.G. is supported by an NIH Pioneer Award DP1AI124669 and an award from the Princeton Catalysis Initiative.

\section{Author contributions}

Experiments were designed and analyzed by S.C., G.V., K.F., and Z.G., experiments were performed by S.C. and G.V., and the paper was written by S.C., G.V., and Z.G.

\section{Additional information}

Supplementary Information accompanies this paper at https://doi.org/10.1038/s41467019-12153-1.

Competing interests: The authors declare no competing interests.

Reprints and permission information is available online at http://npg.nature.com/ reprintsandpermissions/

Peer review information Nature Communications thanks Paul Williams and the other, anonymous, reviewer(s) for their contribution to the peer review of this work. Peer reviewer reports are available.

Publisher's note Springer Nature remains neutral with regard to jurisdictional claims in published maps and institutional affiliations.

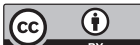

Open Access This article is licensed under a Creative Commons Attribution 4.0 International License, which permits use, sharing, adaptation, distribution and reproduction in any medium or format, as long as you give appropriate credit to the original author(s) and the source, provide a link to the Creative Commons license, and indicate if changes were made. The images or other third party material in this article are included in the article's Creative Commons license, unless indicated otherwise in a credit line to the material. If material is not included in the article's Creative Commons license and your intended use is not permitted by statutory regulation or exceeds the permitted use, you will need to obtain permission directly from the copyright holder. To view a copy of this license, visit http://creativecommons.org/ licenses/by/4.0/.

(c) The Author(s) 2019 